Robert A. Gross, MD, PhD, FAAN, Editor-in-Chief, Neurology $y^{\otimes}$

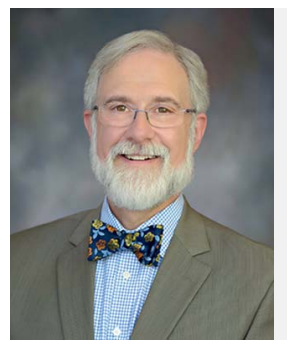

Notable in Neurology this week

This issue features an article that determines that serum creatinine is a candidate biomarker for spinal muscular atrophy progression; another investigates the accuracy of a questionnaire for self-diagnosis of benign paroxysmal positional vertigo. A featured Special Article recommends specific pharmacologic and nonpharmacologic strategies for treating sleep disturbances in children and adolescents with autism spectrum disorder.

\title{
Articles
}

\section{Determinants of quality of life in pediatric- and adult-onset multiple sclerosis}

A central tenet of health care is to improve the quality of life ( $\mathrm{QoL}$ ) of patients. This study found no differences in the QoL of patients with pediatric- and adult-onset multiple sclerosis. A focus on reducing neurologic disability and improving mental health could potentially improve the QoL of patients with multiple sclerosis.

Page 383

\section{White matter hyperintensities and CSF Alzheimer disease biomarkers in preclinical Alzheimer disease}

Small vessel cerebrovascular and Alzheimer disease biomarkers have largely independent and nonsynergistic associations on the risk of mild cognitive impairment. Small vessel cerebrovascular disease, as measured by white matter hyperintensities, predicts clinical symptoms of mild cognitive impairment only when levels of neurodegeneration, as measured by total tau in CSF, are low.

Page 385

\section{Convexity subarachnoid hemorrhage in lobar intracerebral hemorrhage: A prognostic marker}

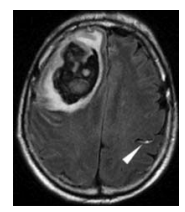

Cerebral amyloid angiopathy (CAA) carries a high, but heterogeneous, risk of intracerebral hemorrhage (ICH). In this cohort of patients with CAA and acute lobar ICH, MRI-detected acute convexity subarachnoid hemorrhage was a predictor of recurrent ICH. This neuroimaging marker could be used in the acute setting to stratify risk of future ICH.

Page 387

From editorialists Smith and Maas: "Because acute ICH is a common context for initial CAA diagnosis and an index event that precipitates important clinical decision-making about antithrombotic medications, their finding has importance."

Page 375

\section{Three-month modified Rankin Scale as a determinant of 5-year cumulative costs after ischemic stroke: An analysis of 11,136 patients in Korea}

A relationship exists between costs and outcomes that is based on the modified Rankin Scale (mRS). Through comparison of a nationwide stroke registry and national claims data, this study 
illustrated that the 3-month mRS assists in predicting long-term costs after stroke. Such predications may help with stroke care-related health economic evaluations.

Page 388

From editorialists Majersik and Woo: "It may seem obvious that cost of stroke is related to outcomes after stroke, but the magnitude of the effect is worth a moment's reflection...therapies that bend the disability curve in even a fraction of patients by shifting outcomes by 1 or more $m R S$ points could have substantial societal economic impact."

Page 377

NB: "Hopkins syndrome: A rare differential diagnosis of neurogenic monomelic amyotrophy," p. e996. To check out other Resident \& Fellow Teaching NeuroImages, point your browser to Neurology.org/N. At the end of the issue, check out the Resident \& Fellow Clinical Reasoning article illustrating the diagnostic process for a patient presenting with slowly progressive bilateral foot drop and ultimately diagnosed with distal hereditary neuropathy. This week also includes a Resident \& Fellow Pearls \& $\mathrm{Oy}$-sters article titled "Primary angiitis of the CNS presenting with recurrent intracranial hemorrhage."

\section{NEW EPISODE}

\section{ค}

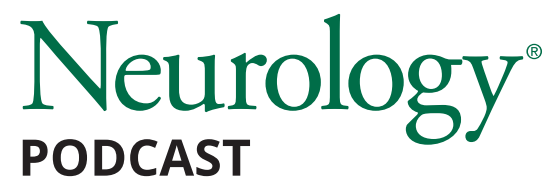

March 3, 2020

\section{Minimal breastmilk transfer of rituximab, a monoclonal antibody used in neurological con- ditions (see the January 2020 issue of Neurology ${ }^{\otimes}$ Neuroimmunology \& Neuroinflammation)}

1. Minimal breastmilk transfer of rituximab, a monoclonal antibody used in neurological conditions

2. What's Trending: Obscure Model Puts a Price on Good Health — and Drives Down Drug Costs

In the first segment, Dr. David Lapides talks with Dr. Riley Bove about her paper on minimal breastmilk transfer of rituximab, a monoclonal antibody used in neurological conditions. In the second part of the podcast, Dr. Jason Crowell speaks with Denise Roland of the Wall Street Journal about her story on an obscure model driving down drug costs. You can read the paper online here: https://www.wsj.com/articles/obscure-model-puts-a-price-on-good-healthanddrives-down-drug-costs-11572885123.

Disclosures can be found at Neurology.org.

No CME this week: Interviews based on articles from Neurology ${ }^{\circledR}$ Clinical Practice, Neurology ${ }^{\circledR}$ Genetics, and Neurology: Neuroimmunology \& Neuroinflammation are excluded from the CME program. 


\section{Neurology}

\section{Spotlight on the March 3 issue}

Robert A. Gross

Neurology 2020;94;371-372

DOI 10.1212/WNL.0000000000009031

This information is current as of March 2, 2020

\section{Updated Information \&} Services

Permissions \& Licensing

Reprints including high resolution figures, can be found at: http://n.neurology.org/content/94/9/371.full

Information about reproducing this article in parts (figures,tables) or in its entirety can be found online at:

http://www.neurology.org/about/about_the_journal\#permissions

Information about ordering reprints can be found online:

http://n.neurology.org/subscribers/advertise

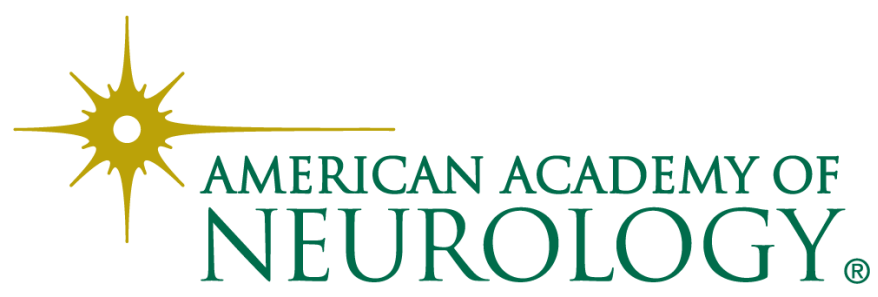

